University of South Florida

DIGITAL COMMONS

Digital Commons @ University of

@ UNIVERSITY OF SOUTH FLORIDA

South Florida

March 2020

\title{
Using Generalization-Enhanced Behavioral Skills Training to Teach Poison Safety Skills to Children with Autism
}

Ellie Morosohk

University of South Florida

Follow this and additional works at: https://digitalcommons.usf.edu/etd

Part of the Social and Behavioral Sciences Commons

\section{Scholar Commons Citation}

Morosohk, Ellie, "Using Generalization-Enhanced Behavioral Skills Training to Teach Poison Safety Skills to Children with Autism" (2020). USF Tampa Graduate Theses and Dissertations.

https://digitalcommons.usf.edu/etd/8262

This Thesis is brought to you for free and open access by the USF Graduate Theses and Dissertations at Digital Commons @ University of South Florida. It has been accepted for inclusion in USF Tampa Graduate Theses and Dissertations by an authorized administrator of Digital Commons @ University of South Florida. For more information, please contact digitalcommons@usf.edu. 
Using Generalization-Enhanced Behavioral Skills Training to Teach Poison Safety Skills to Children with Autism

by

Ellie Morosohk

\begin{abstract}
A thesis submitted in partial fulfillment of the requirements for the degree of Master of Science in Applied Behavior Analysis

Department of Child and Family Studies College of Behavioral and Community Sciences University of South Florida
\end{abstract}

\author{
Major Professor: Raymond Miltenberger, Ph.D., BCBA-D \\ Catia Cividini-Motta, Ph.D., BCBA-D \\ Kimberly Crosland, Ph.D., BCBA-D
}

Date of Approval:

March 5, 2020

Keywords: In situ assessments, Pills, Tangible reinforcers

Copyright $($ C 2020, Ellie Morosohk 


\section{ACKNOWLEDGMENTS}

First, I would like to thank my family for their encouragement through graduate school. Thank you to my mom for her unwavering support, Shira for always pushing me to be better, and my dad for shaping me into the person I am today. I would like to express my gratitude to my major advisor Dr. Miltenberger for guiding me through the thesis process. Thank you to my committee members, Dr. Crosland and Dr. Cividini-Motta for offering their feedback. Finally, I would like the thank Zoe Hay for your help with data collection throughout this process. 


\section{TABLE OF CONTENTS}

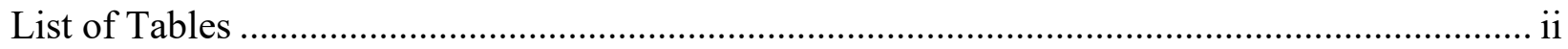

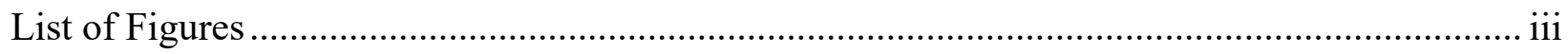

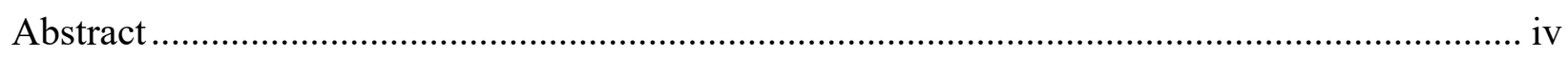

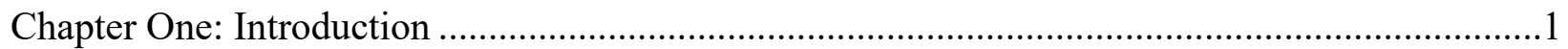

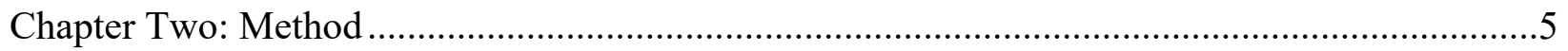

Participants and Setting ........................................................................................

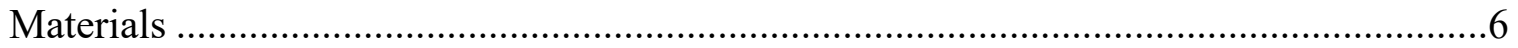

Target Behaviors and Data Collection ......................................................................

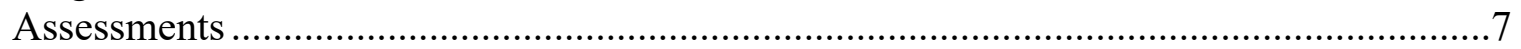

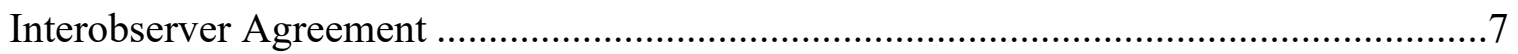

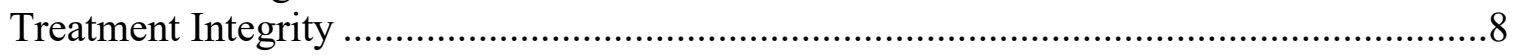

Social Validity Assessment.................................................................................

Experimental Design and Procedures ……………......................................................

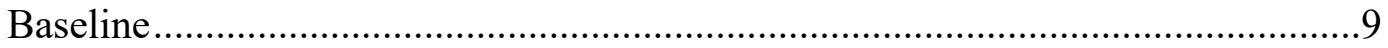

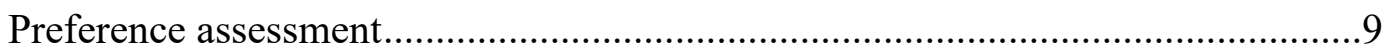

Generalization-enhanced BST .....................................................................

Follow-up ...............................................................................................11

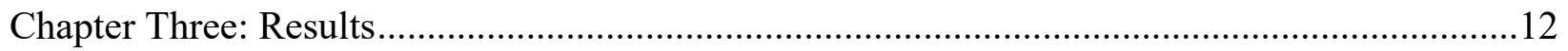

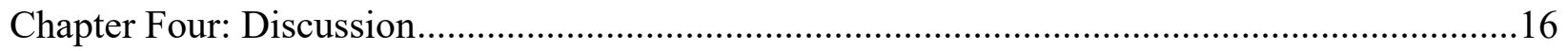

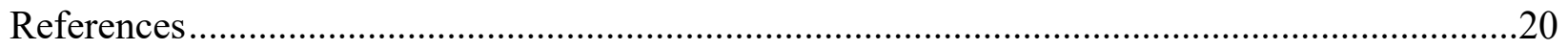

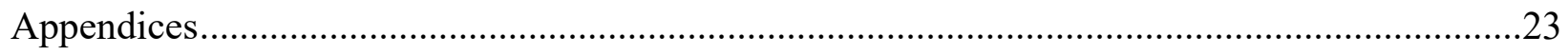

Appendix A: Treatment Integrity Checklist ……………........................................24

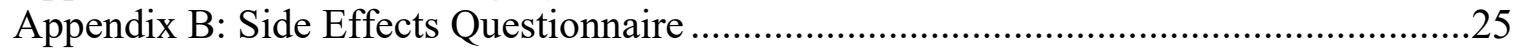

Appendix C: Social Validity Questionnaire …………….......................................26

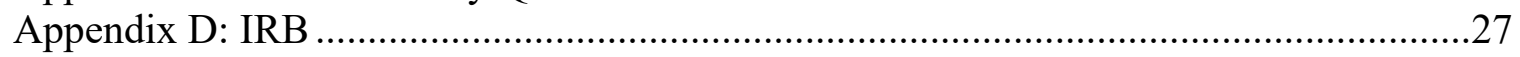




\section{LIST OF TABLES}

Table 1: Assessment conditions are described for in situ assessments for each participant.....15 


\section{LIST OF FIGURES}

Figure 1: The safety skills score for each participant are depicted for baseline, intervention,

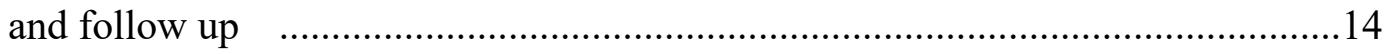




\begin{abstract}
It is important for children to learn safety skills, especially involving dangerous stimuli such as medicine that can be found in almost every household. This study examined a generalizationenhanced behavioral skills training package to teach children with autism poison safety skills. Three children ages 4- to 10-years-old received generalization enhanced BST to teach the safety skills required when they found pills. In situ assessments were conducted both in home and in the clinic to examine whether the skills generalized to the natural setting. All children engaged in the safety skills following training. The results of this study indicated that generalization enhanced BST is an effective method of teaching poison safety skills to children with autism.
\end{abstract}




\section{CHAPTER ONE:}

\section{INTRODUCTION}

Children's safety can be at risk due to different threats that can be found in their natural environment. One major safety threat that can be found in every household is poisonous substances. In the United States, each day more than 300 children are treated in the emergency room, and two children die because of poison (Centers for Disease Control and Prevention [CDC], 2016). According to the CDC (2016), poisonous substances are not just substances that are clearly labeled as dangerous, but any cleaners or medicines that could be harmful if ingested inappropriately. Clearly this is a safety threat that needs to be addressed. Recommendations for keeping children safe from poison threats focus heavily on keeping the poison out of the reach of children and locking it up (World Health Organization, 2010). Although this idea of child proofing and keeping the environment safe is important, it does not always work. Adults can make mistakes and leave dangerous substances out that they don't mean to, or might not realize that something like medicine is harmful. Therefore, it is imperative to teach children the skills to keep themselves safe. Additionally, children with developmental disorders are at higher risk for accidental poisoning (Sinclair \& Xiang, 2008), so teaching poison safety skills to children with autism is especially important.

One of the most common methods used to teach safety skills is behavioral skills training consisting of instructions, modeling, rehearsal, and feedback (BST, Miltenberger, 2016). BST has been used to teach typically developing children of different ages firearm safety skills (e.g., Himle, Miltenberger, Flessner, \& Gatheridge, 2004; Miltenberger et al., 2004) and abduction 
prevention skills (e.g., Johnson et al., 2005). The results of these studies demonstrate that about half of the children learned the skills from BST alone, and the other half required in situ training (IST). Therefore, some modifications may be required to make BST a more effective training procedure.

BST has been used in interventions to teach safety skills to children diagnosed with autism. Ledbetter-Cho et al. (2016) examined BST as an intervention to teach abduction prevention skills to children with autism. In this study, BST was enhanced with rehearsals in the natural environment with strangers playing the role of abductors. Furthermore, feedback was delivered in the natural environment so it was similar to in situ training. This study had successful results with the enhanced BST, and all participants learned the skills. Studies have also investigated BST to teach multiple safety skills to children diagnosed with autism. Rossi, Vladescu, Reeve, and Gross (2017) used BST to teach safety skills from three categories, fire safety, poison prevention, and firearms avoidance to children with autism. They modified BST to include a tangible reinforcer for one participant. Additionally, Summers et al. (2011) used BST with least to most prompting to teach the household safety skills of responding to doorbells and household chemicals to children with autism. These studies had successful results indicating that BST with additional procedures might be an appropriate method to teach safety skills to children with autism.

Currently, there is limited research involving teaching poison safety skills. Dancho, Thompson, and Rhoades (2008) examined the use of group BST methods to teach poison safety skills involving medication to 15 typically developing preschool children. Group BST was not found to be successful. Video modeling has also been examined as a method to teach poison safety skills using medication (King \& Miltenberger 2016). This study was conducted with 6- 
year-old children diagnosed with autism. However, video modeling was not found to be a successful method of teaching the safety skills to this population. Although group BST did not work in Dancho et al., modified BST has been implemented successfully with individual children to teach poison safety skills using household chemicals as the stimuli (Rossi et al., 2017; Summers et al., 2011). Most recently, a modified BST intervention involving most-to-least prompting was implemented to teach poison safety skills in the presence of medication, to children with autism (Petit-Frere, \& Miltenberger, 2019). This study showed promising results that BST if modified appropriately can be an effective intervention to teach these safety skills to children with autism. Overall, more research is required in the field of poison safety to find the most effective, but least intrusive intervention to teach the safety skills children require to keep themselves safe in the presence of poison.

Although some modifications of BST to teach safety skills have been successful, there is still more work to be done. One intervention component that could be added to BST is tangible reinforcement in addition to praise to strengthen the responses (e.g. Orner \& Miltenberger, 2019; Rossi et al., 2017). For example, Orner and Miltenberger (2019) found that when teaching gun safety to children diagnosed with autism using small scale simulation, one of the three participants did not obtain the skills through simulation training or in situ training and required tangible reinforcement to learn the skills. King and Miltenberger (2017) reported similar findings when teaching poison prevention skills to children with autism. Therefore, some children with autism may require tangible reinforcers rather than praise alone to learn safety skills. Providing preferred tangible reinforcers in addition to praise is not difficult or time consuming, and could make a large difference in the way that children with autism learn critical safety skills. 
Methods to enhance generalization can also be added to BST to make it more effective. In situ training is one generalization enhancing method shown to be effective for a number of safety skills (Godish, Miltenberger, \& Sanchez, 2017; Himle et al., 2004; Miltenberger et al., 2004; Morgan \& Miltenberger, 2017). However, in situ training is time and resource intensive and is usually used after BST fails. It is more efficient to incorporate generalization methods into BST than to add in situ training later. Taylor and Harris (1995) incorporated generalization methods into training by incorporating new rooms, new people and new stimuli. Including these components helped the skills generalize outside of the training setting. Other studies have used generalization methods of fading the researcher out of the training situation as participants learn the skills (Bergstrom et al., 2012; Petit-Frere \& Miltenberger, 2019). Incorporating these generalization methods into training may help the participant use the skills outside of the training situation when they are most relevant. However, more research is needed to establish the effectiveness of these methods. Therefore, the purpose of this study is to examine whether adding tangible reinforcement and incorporating generalization methods with traditional behavioral skills training will be effective for teaching poison safety skills to children with autism. 


\section{CHAPTER 2:}

\section{Participants and Setting}

\section{METHOD}

Participants in this study were three boys ages 4 - to 10- years old, diagnosed with autism spectrum disorder (ASD) recruited from an ABA clinic. All of the participants had vocal repertoires and the ability to leave a room and tell an adult that they found pills. This was assessed by showing the child pills and asking what it was. The child was required, at minimum, to answer in one word, pills, but speaking in a full sentence was preferred if the child had a more advanced repertoire. An assessment was conducted examining participants' abilities to observe an event occur in one room and report the event to a caregiver in a different location. This assessment was carried out by having the child play with a particular toy in one room. They were then asked to enter a different room and tell an adult what they were playing with. In addition, all children participating in this study had no prior poison safety skills training. Pseudonyms are used to discuss the participants, and information about the participants diagnosis and routine is based on parent report.

Logan was a 6-year-old boy diagnosed with high functioning ASD and severe behavior disorder. He had good verbal skills and spoke in full sentences. He attended kindergarten for 6.5 hr a day, 5 days a week and he was in a classroom for typically developing children. Logan had been receiving ABA therapy for one year at the beginning of the study for an average of $25 \mathrm{hr}$ per week. 
Connor was a 10-year-old boy diagnosed with ASD. He was in fifth grade and attended school 5 days a week for $6.25 \mathrm{hr}$ each day. He had strong verbal abilities and communicated in full sentences. He had been receiving ABA services for 3 months at the beginning of the study. For two of the months he was attending one session a week for $3 \mathrm{hr}$, and in the last month of the study he started attending two sessions a week for a total of $6 \mathrm{hr}$.

Samuel was a 4-year-old boy diagnosed with ASD level 2 which indicates a moderate severity level. He attended preschool for $4 \mathrm{hr}$ in the morning and therapy in the afternoons. $\mathrm{He}$ received $25 \mathrm{hr}$ of therapy a week, $22 \mathrm{hr}$ consisted of ABA therapy in a clinic, and the other $3 \mathrm{hr}$ consisted of speech therapy, occupational therapy and physical therapy. Samuel had strong echoic and manding repertoires (he could repeat what he heard and ask for things he wanted) but his intraverbal repertoire was not as developed, although he did communicate in full sentences.

Assessments for this study were conducted both in the clinic and in home. The training sessions took place mostly in the clinic and involved multiple rooms, including the bathroom and kitchen where pills were found. In the home, the bathrooms, kitchen, living room, and bedrooms were utilized. The location where the pills were found was based on availability and changed each session.

\section{Materials}

Empty pill capsules were used in this study to imitate real medications. Pill capsules were used rather than candy to make the situation more realistic, and minimize the risk that consumption could be reinforcing. The pill capsules were placed in pill bottles, pillboxes, and clear plastic bags. The pill capsules were size 0 pills that were blue and green. 


\section{Target Behaviors and Data Collection}

The target behavior involved three components: not touching the pills or containers defined as no part of their body making contact, leaving the room within $10 \mathrm{~s}$ of seeing the pills, and telling an adult by vocally saying, "I found pills" or just "pills." The data were scored on the

following 4-point scale: $0=$ touched the pills or the container; $1=$ did not leave the room, but did not touch the pills or container; $2=$ did not touch the pills and left the room or did not touch the pills and told an adult after yelling for the adult to come to the room; $3=$ did not touch the pills or containers, left the room within $10 \mathrm{~s}$ and told an adult about the pills within $10 \mathrm{~s}$ of contacting the adult. If the child touched the pill container, the researcher also recorded any subsequent behaviors such as opening or trying the open the container and manipulating or consuming the pills.

\section{Assessment}

In situ assessments were conducted throughout all phases of the study both in the home and in clinic. To begin the in situ assessments, the participants were unaware that the researcher was present in the situation and either the parents or a therapist instructed the child to go to a room where the pills had been placed clearly in sight. Hidden cameras were positioned ahead of time to observe the child's execution of the target behaviors once he had entered the room. If the child did not leave the room within $30 \mathrm{~s}$, the teacher or parent entered the room and casually removed the medication (from where it was placed or from the child's hands) without commenting. If a child left the room and reported the medication, the parents or teacher thanked the participant. 


\section{Interobserver Agreement}

To calculate interobserver agreement (IOA), a second observer independently scored videos from the in situ assessments. The second observer used the 4-point scale to score $36 \%$ of videos from baseline and intervention and $33 \%$ of videos from follow up assessments. If both observers indicated that a target behavior had occurred, this was an agreement. To calculate IOA, the number of agreements on the three behaviors was divided by agreements plus disagreements and then multiplied by 100 . IOA was $100 \%$.

\section{Treatment Integrity}

Treatment integrity (TI) data were collected based on the percentage of steps the researcher completed correctly during generalization-enhanced BST (hereafter referred to as BST) sessions. A task analysis of the steps required for BST sessions was developed to collect TI data (see Appendix A). The percentage of TI was calculated by dividing the number of steps completed correctly by the total number of steps and then multiplying by 100 . Treatment integrity was scored by the principle investigator on $100 \%$ of BST sessions. Treatment integrity IOA was scored by a second observer on $25 \%$ of BST sessions, and was scored based on videos taken during the sessions. IOA was calculated by dividing the number of agreement between the two observers on the task analysis steps and then multiplying by 100 . Treatment integrity data was $100 \%$. IOA on TI data was $100 \%$.

\section{Social Validity Assessment}

Following participation in this research study, parents were asked to complete two questionnaires (Appendices B and C) to provide their opinion of the study and indicate any side effects they observed in their child. This questionnaire targeted the parents' opinion on the 
effectiveness of the intervention and any changes that they observed in their children that could be attributed to participation in the study.

\section{Experimental Design and Procedures}

A nonconcurrent multiple baseline design across participants was utilized to evaluate the effects of the BST across the participants. The study began with baseline in situ assessments for all participants both in the home and in the clinic. This was followed by BST delivered to one participant at a time. Mastery criterion was a score of 3 on three consecutive assessments. At least 1 week after treatment and assessments were completed, follow up data were collected.

Baseline. In situ assessment were conducted both in the clinic and in home. The child was unaware that his or her behavior was being assessed, and no training or feedback was provided based on the performance during the assessment. Baseline in situ assessments were conducted with each child until the data were stable.

Preference assessment. Intervention began by conducting a single trial preference assessment with each participant. In this preference assessment, the child was presented with an array of four to six items based on report from parents and therapists on toys they enjoyed. The child was told to "pick one" and then engage with that toy. The tangible that they selected from the array was used as the reinforcer for that training session. This single trial preference assessment was conducted prior to each BST training session. In this preference assessment, Logan chose a bouncy ball, Connor chose trains, and Samuel chose a toy phone and a remote. During in situ assessments, the most recent tangible reinforcer that was selected was used, because a preference assessment could not be conducted because the participant is unaware of the researchers presence during in situ assessments. 
Generalization-enhanced BST. The intervention incorporated all components of traditional BST: instructions, modeling, rehearsal and feedback. Participants received an individual BST session with the researcher. To begin the session the researcher told the child about the dangers of eating pills they find and the types of containers in which they can find pills. The researcher then described the three safety skills that they should engage in when they find pills, not touching the pills, leaving the room and telling an adult. The researcher then demonstrated these skills when finding pills. The researcher demonstrated by walking into a room, finding pills in one of the containers, and then running out of the room to tell a staff member. The demonstration was repeated with each of the three pill containers in three different locations. Next, the researcher had the participant practice the skills. Practice occurred as the participant found the pills in each of the three containers in three different locations. BST was enhanced with tangible reinforcers for engaging in the correct safety skills. Tangible reinforcers were used in addition to praise for correct performance and verbal feedback was used for incorrect performance. Rehearsals continued until unprompted correct behavior occurred in the presence of each type of pill container. Generalization strategies were incorporated in the BST training session by practicing in multiple locations, and fading the researcher out of the room during rehearsals. Fading was accomplished initially using a binary fading system. First the researcher was with the participant, then a role play was set up that required the participant to enter the room with the pills alone and then run from the room to find the staff to report the pills. If the child did not perform the safety skills the first time the researcher left the room, a fading procedure involving moving further away from the child after each trial was utilized. First the researcher was standing next to the child, then the researcher moved $1 \mathrm{~m}$ away, then $1.6 \mathrm{~m}$ away, then $2.5 \mathrm{~m}$ away and then completely exited the room. 
An in situ assessment was conducted in the clinic within 7 days following the BST session. If the safety skills were performed, in situ assessments were implemented both in the home and in the clinic until the participant achieved three consecutive scores of 3 . If all three safety skills were not performed, a booster training session was provided. Only one booster session was needed.

Follow-up. In situ assessments were conducted 1 to 8 weeks after the final assessment had been conducted. No training or feedback was involved, however tangible reinforcers were provided following a correct response so the response was not placed on extinction conditions. 


\section{CHAPTER THREE:}

\section{RESULTS}

All participants exhibited the safety skills during in situ assessments following generalization enhanced BST. Logan and Connor required one training session to exhibit the safety skills and meet mastery criterion, and Samuel required the initial BST session and one booster session before he exhibited the safety skills. In baseline, Logan touched the pill containers during three in situ assessments, consisting of two in clinic assessments and one in home assessment. In the first two assessments when the pills were placed in a clear plastic bag and pillbox he picked up the pill container but did not open it, and on the third assessment with the pill bottle he opened the container but did not try to eat the pills. Logan's individual BST session was $25 \mathrm{~min}$ long and 10 rooms were used to practice the safety skills. Following BST, Logan engaged in all safety skills in four consecutive assessments both in clinic and in home. After the final in situ assessment, follow up assessments were conduct 11 days later in clinic and 8 weeks later in home. He continued to demonstrate the correct safety skills in follow up in situ assessments.

Connor had five in situ assessments in baseline which all took place in clinic. He touched the pill container one time when the pills were in a clear plastic bag. During this assessment he took the pills out of the bag and took them apart. In the other four assessments he did not touch the pills, but stayed in the area and engaged in the activity he was told to. Connor's BST session was 29 min long and five rooms were used to practice the safety skills. After his BST session, Connor completed all safety skills on three consecutive in situ assessments. He had one follow 
up assessment 2 weeks after the final post-intervention assessment and he continued to demonstrate all safety skills.

Samuel had five baseline in situ assessment in the clinic and one baseline assessment in home. He touched the pill containers in three of the assessments, and did not touch the pill container but stayed in the room for the other three baseline assessments. The initial BST session conducted with Samuel was in the clinic and lasted $30 \mathrm{~min}$. The booster session was conducted in his home and was 19 min long. In total he received less than 49 min of individual BST to demonstrate the safety skills. After his initial BST session he scored a 2 on an in situ assessment in the clinic. In this assessment he did not touch the pills, told himself to walk away but did not actually leave, and yelled to his therapist that he found pills from where he was. After the booster BST session he scored a 3 on three consecutive in home assessments. However, in Samuel's 6 week follow up assessment he touched the pills to move them out of the way so he could play.

Data on social validity were collected at the end of the study from questionnaires the parents completed. Questions were ranked from 1 strongly disagree to 5 strongly agree, and averaged across parents. Parents were happy with their participation in the study (5), and said they would recommend the intervention to other families (5). Parents also reported that their children are more cautious around medicine (4), and their children were not upset by participating in the study (1). Samuel's parents reported that they would like to examine the poison safety skills with syrups (liquid medication), and Logan's parents reported that they would be interested to see the safety skills demonstrated with pills that are not placed in a container. 


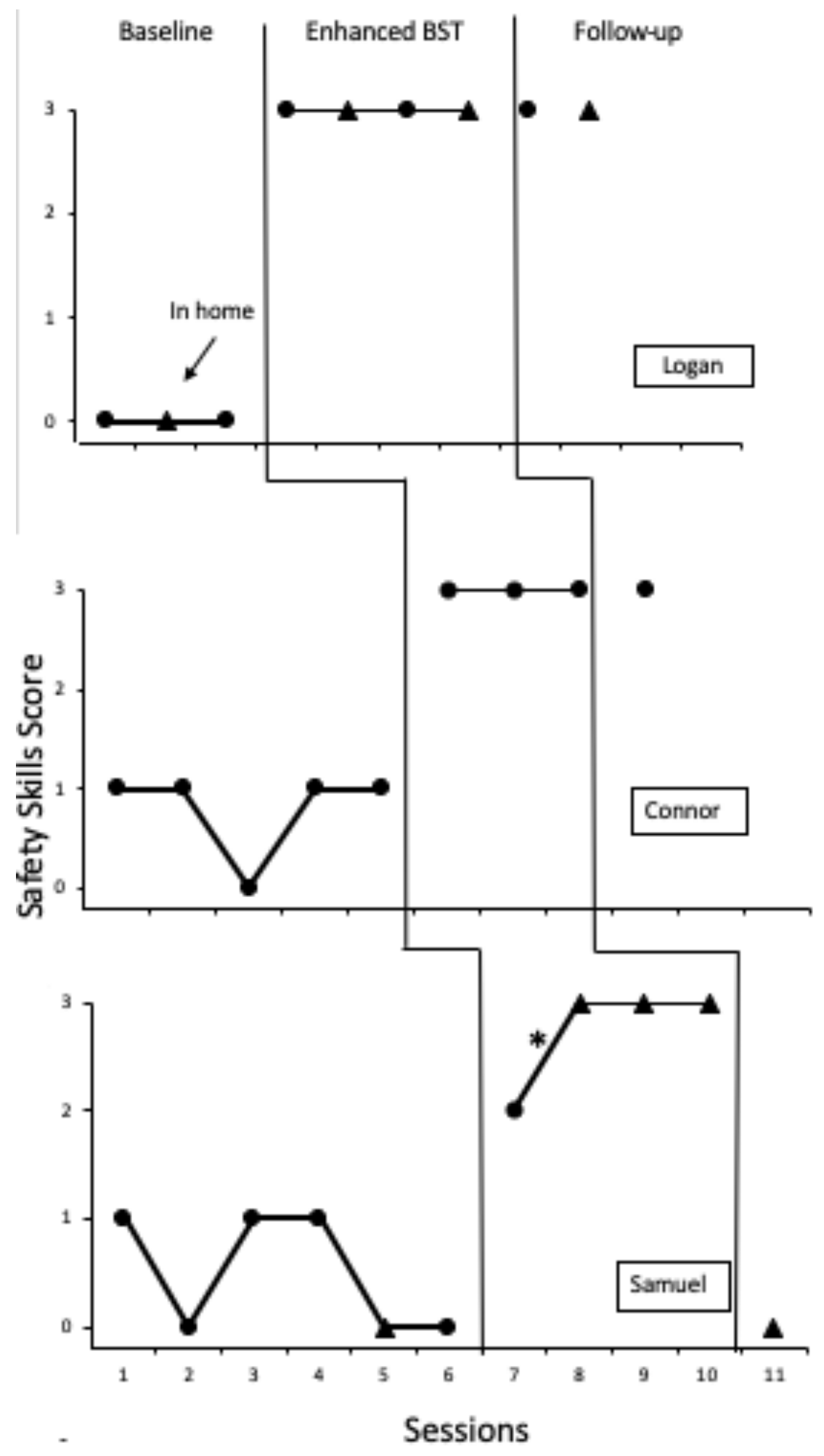

Figure 1. The safety skills score for each participant are depicted for baseline, intervention, and follow up. An Asterisk indicates a booster BST session occurred. 
Table 1. Assessment conditions are described for in situ assessments for each participant

Logan

\begin{tabular}{l|l|l|l|}
\hline Baseline & Pill Color/ Container & Location & Statement \\
\hline & Ziplock/ Green & Kitchen & Go eat your snack \\
\hline & Pill box/ Blue & Kitchen & Go play with the decorations \\
\hline & Pill bottle/ Green & Therapy room & Go play with your legos \\
\hline BST & Pill box/ Blue & Group room & Go do an art project \\
\hline & Ziplock/ Blue & Kitchen & Go eat your ice cream \\
\hline & Pill bottle/ Green & Office & Go watch a video \\
\hline & Pill box/ Green & Family room & Go eat your candy \\
\hline Follow up & Ziplock/ Green & Kitchen & Go eat your snack \\
\hline & Pill box/ Blue & kitchen & Go play with your aligator toy \\
\hline
\end{tabular}

Connor

\begin{tabular}{|l|l|l|l|}
\hline & Pill Color/ Container & Location & Statement \\
\hline & Pill box/ Blue & Therapy room & Go play with your trains \\
\hline & Pill bottle/ Green & Play room & Go play with marble run \\
\hline & Ziplock/ Blue & Kitchen & Go eat your dinner \\
\hline & Pill bottle/ Blue & Therapy room & Go play the drums \\
\hline & Pill box/ Blue & Play room & Go play with bubbles \\
\hline BST & Ziplock/ Green & Bathroom & Go wash your hands \\
\hline & Pill bottle/ Blue & Group room & Go play with drums \\
\hline Follow up & Pill box/ Green & Kitchen & Go eat your dinner \\
\hline Ziplock/ Green & Therapy room & Go play with your putty \\
\hline
\end{tabular}

Samuel

\begin{tabular}{|l|l|l|l|}
\hline & Pill Color/ Container & Location & Statement \\
\hline Baseline & Ziplock/ Green & Playroom & Go play with the letters \\
\hline & Pill bottle/ Blue & Bathroom & Go wash your hands \\
\hline & Pill box/ Green & Therapy room & Go play with your fish \\
\hline & Pill bottle/ Green & Kitchen & Go eat your chips \\
\hline & Ziplock/ Blue & Living room & Go play with your trains \\
\hline & Pill box/ Blue & Bathroom & Go wash your hands \\
\hline & Ziplock/ Green & Therapy room & Go play the game \\
\hline & Pill box/ Blue & Playroom & Go sit at your table \\
\hline & Pill bottle/ Green & Kitchen & Go read your book \\
\hline & Ziplock/ Blue & Living room & Go play with your toy \\
\hline & Pill box/ Blue & Kitchen & Go play with the beads \\
\hline Follow up & & &
\end{tabular}




\section{CHAPTER 4}

\section{DISCUSSION}

This study examined the effects of generalization enhanced BST to teach poison safety skills to children diagnosed with autism. The results indicated that generalization enhanced BST was an effective training method for the three participants involved in this study. All three children exhibited the safety skills required to keep themselves safe when they found pills during in situ assessment simulating real scenarios in the natural environment. Logan and Connor required one BST session and Samuel required two BST sessions to exhibit the safety skills to mastery criterion. Logan and Connor maintained the safety skills in two week follow up assessments, and Logan maintained the skills in an 8 week follow up. However, Samuel did not maintain the safety skills in a 6 week follow up assessment. None of the participants required in situ training, which would have been the next phase if the safety skills were not exhibited during in situ assessments.

This study expanded on the currently limited literature involving teaching poison safety skills. Previously, both group BST and video modeling were found to be ineffective methods of teaching poison safety skills to children (Dancho et al., 2008: King \& Miltenberger, 2017). In both of these studies, in situ training or incentives were ultimately required to promote the generalization of the safety skills to in situ assessments. Previously, in safety skills studies, tangible reinforcers have been used only after BST and IST have been ineffective (King \& Miltenberger 2017: Orner \& Miltenberger, 2019). However, as this study demonstrated, it was an easy modification to incorporate tangible reinforcers into the training session. IST is time 
intensive and requires the researcher's presence in the assessment situations, so it should be avoided if possible. By using tangible reinforcers from the start, instead of as a last resort, this study was able to avoid using intrusive in situ training.

There has been greater success in teaching poison safety skills using individualized BST with modifications. Recent research has focused on how to modify the original BST training protocol to improve results and make more intrusive training methods less necessary. Most recently, Petit-Frere and Miltenberger (2019) demonstrated successful results using BST and system of least prompts to teach poison safety skills to children with autism. The current research expanded on this by incorporating generalization methods and tangible reinforcers into the original BST sessions to further maximize the effectiveness of the training session, and ultimately eliminate the need for in situ training. Therefore, the current study demonstrates two components that are important modifications to traditional BST.

It is interesting to note that in this study, two of the three participants engaged in self talk while performing the safety skills following BST. Both Logan and Samuel would engage in a behavior chain by telling themselves each step before performing it when they found the pills. For example, when Logan saw the pills he would say “stop, there are pills, don't touch, walk away" and then leave to tell either his therapist or parent. It is also important to note that Samuel liked having small toys to hold in his hands and throughout the study, he wanted to hold the pills. Therefore, after BST there were competing reinforcers between touching the pills or completing the steps he knew and the praise and tangible reinforcer he would then receive. In multiple assessments following BST he completed all the safety steps but instead of reporting that he found pills, he left the room and asked his parents for the pills. This was counted as a correct response because he was alerting the adult to the presence of the pills and completing all relevant 
steps. Ultimately, he did complete the safety skills during the in situ assessments following one booster session. So the praise and tangible reinforcer provided strong enough reinforcement to compete with the reinforcement that resulted from picking up and holding the pills. It should also be noted that throughout the study, none of the participants attempted to ingest the pills or put them in their mouth. In the instances when they opened the containers they either played with the pills or tried to take them apart.

A few limitations were identified in the current research study. First, Connor's assessments took place only in the clinic so generalization of the safety skills to the home was not assessed. However some assessments after BST took place in rooms where training did not occur, so generalization to novel spaces in the clinic was examined. Additionally, Samuel received the first BST session in the clinic, however the booster BST session took place in home and the remainder of in situ assessment took place in the home. Therefore, there was limited generalization assessed with him as well.

Future studies could replicate this study using generalization enhanced BST with tangible reinforcers to teach other safety skills such as gun safety and abduction safety. Also, future research could focus on using generalization enhanced BST to teach poison safety skills to children with autism who are lower functioning than the participants in the current study. All children in this study had verbal repertoires and the ability to report an event in one room to someone in a different location. It would be important to assess whether generalization enhanced BST is capable of teaching these important safety skills to lower functioning children. There is also limited research on teaching poison safety skills to typically developing children, so research could examine what methods are most effective and efficient at teaching that population. Future research could assess generalization in additional settings like the school and 
the community in addition to the home assessments utilized in this study. Finally, based on report from parents on social validity assessments, future research could examine safety skills training with liquid medication in addition to pills, and pills that are not placed in a container.

In conclusion, the generalization enhanced BST package evaluated in this study was found to be effective in teaching three children diagnosed with autism the safety skills required to keep themselves safe around pills. Additionally, skills maintained for two of the three participants. Incorporating generalization methods and tangible reinforcers into BST training sessions appears to be an effective and efficient way of disseminating these important safety skills, and should be explored further. 


\section{REFERENCES}

Bergstrom, R., Najdowski, A. C., \& Tarbox, J. (2012). Teaching children with autism to seek help when lost in public. Journal of Applied Behavior Analysis, 45, 191-195.

Centers for Disease Control and Prevention. (2016). Poison prevention. Retrieved from http://www.cdc.gov/safechild/poisoning/index.html

Dancho, K. A., Thompson, R. H., \& Rhoades, M. M. (2008). Teaching preschool children to avoid poison hazards. Journal of Applied Behavior Analysis, 41, 267-271.

Godish, D., Miltenberger, R., \& Sanchez, S. (2017). Evaluation of video modeling to teach abduction prevention skills to children with autism spectrum disorder. Advances in Neurodevelopmental Disorders, 1, 168-175.

Himle, M. B., Miltenberger, R. G., Flessner, C., \& Gatheridge, B. (2004). Teaching safety skills to children to prevent gun play. Journal of Applied Behavior Analysis, 37, 1-9.

Johnson, B. M., Miltenberger, R. G., Egemo-Helm, K., Jostad, C. M., Flessner, C., \& Gatheridge, B. (2005). Evaluation of behavioral skills training for teaching abduction prevention skills to young children. Journal of Applied Behavior Analysis, 38, 67-78.

King, S. \& Miltenberger, R. (2017). Evaluation of video modeling to teach children with autism to avoid poison hazards. Advances in Neurodevelopmental Disorders, 1, 221-229.

Ledbetter-Cho, K., Lang, K., Davenport, K., Moore, M., Lee, A., O-Reilly, M., Watkins, L., \& 
Falcomata, T. (2016). Behavior skills training to improve the abduction-prevention skills of children with autism. Behavior Analysis in Practice, 9, 266-270.

Miltenberger, R. G. (2016). Behavior Modification: Principles and Procedures (6 $6^{\text {th }}$ ed.) Belmont, CA: Thompson Wadsworth.

Miltenberger, R. G., Flessner, C., Gatheridge, B., Johnson, B., Satterlund, M., \& Egemo, K. (2004). Evaluation of behavioral skills training to prevent gun play in children. Journal of Applied Behavior Analysis, 37, 513-516.

Morgan, K., \& Miltenberger, R. G. (2017). Evaluation of video modeling and in situ training to teach firearm avoidance skills to individuals with autism spectrum disorder. Advances in Neurodevelopmental Disorders, 1, 122-128.

Orner, M \& Miltenberger, R. G. (2019). Evaluating small scale simulation training for teaching firearm safety skills to children with ASD. Unpublished manuscript.

Petit-Frere, P. \& Miltenberger, R. G. (2019). An evaluation of a modified behavioral skills training procedure for teaching poison prevention skills to children with developmental disabilities. Unpublished manuscript

Rossi, M. R., Vladescu, J. C., Reeve, K. F., \& Gross, A. C. (2017). Teaching safety responding to children with autism spectrum disorder. Education and Treatment of Children, 40 (2), 187-208. 
Sinclair, S. \& Xiang, H. (2008). Injuries among US children with different types of disabilities. American Journal of Public Health, 98, 1510-1516.

Summers, J., Tarbox, J., Findel-Pyles, R. S., Wilke, A. E., Bergstrom, R., \& Williams, W. L. (2011). Teaching two household safety skills to children with autism. Research in Autism Spectrum Disorders, 5, 629-632.

Taylor, B. A., \& Harris, S. L. (1995). Teaching children with autism to seek information: acquisition of novel information and generalization of responding. Journal of Applied Behavior Analysis, 28, 3-14.

World Health Organization. (2010). Healthy environments for healthy children: Key messages for action. Retrieved from http://apps.who.int/iris/bitstream/10665/44381/1/9789241599887_eng.pdf 
APPENDICIES 


\title{
Appendix A: Treatment Integrity Checklist
}

\author{
Generalization Enhanced BST
}

\begin{tabular}{|l|l|}
\hline \multicolumn{1}{|c|}{ Skill } & \multicolumn{1}{|c|}{ Completed Correctly Y/N } \\
\hline Providing instructions & \\
\hline Modeling the skill & \\
\hline Rehearsing with the child & \\
\hline Providing verbal feedback & \\
\hline $\begin{array}{l}\text { Providing tangible reinforcement contingent on } \\
\text { completing all steps correctly }\end{array}$ & \\
\hline $\begin{array}{l}\text { Completing training sessions in different rooms. } \\
\text { This includes training sessions occurring in at } \\
\text { least three different rooms Move to a new room } \\
\text { when the child enters one room and performs all } \\
\text { safety skills independently. }\end{array}$ & \\
\hline $\begin{array}{l}\text { Child encountering all three types of pill } \\
\text { containers. Child must perform the safety skills } \\
\text { the presence of all containers }\end{array}$ & \\
\hline $\begin{array}{l}\text { Fading researcher out. By the end of training, } \\
\text { the child will complete all safety skills with the } \\
\text { researcher out of sight. }\end{array}$ & \\
\hline
\end{tabular}


Appendix B: Side Effect Questionnaire

Side Effects Questionnaire

$\begin{array}{ccccc}1 & 2 & 3 & 4 & 5 \\ \text { Strongly Disagree } & \text { Disagree } & \text { Neutral } & \text { Agree } & \text { Strongly Agree }\end{array}$

1) My child is more

cautious around

1

2

3

4

medicine

2) My child has

been upset

1

2

3

4

5

after participation

3) Have you seen any other changes in your child? 


\section{Appendix C: Social Validity Questionnaire}

Social Validity Questionnaire

$\begin{array}{ccccc}1 & 2 & 3 & 4 & 5 \\ \text { Strongly Disagree } & \text { Disagree } & \text { Neutral } & \text { Agree } & \text { Strongly Agree }\end{array}$

1) How happy are

you with your

1

2

3

4

participation in

this study

2) I would recommend

this intervention

1

2

3

4

5

to other families

3) Do you have any other comments about your participation in this study? 


\section{Appendix D: IRB}

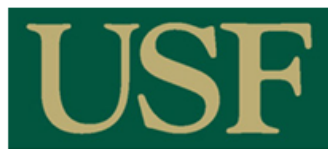

UNIVERSITY OF

SOUTH FLORIDA
RESEARCH INTEGRITY AND COMPLIANCE

Institutional Review Boards, FWA No. 00001669

12901 Bruce B. Downs Blvd., MDC035 • Tampa, FL 33612-4799

(813) $974-5638$ • FAX (813) 974.7091

July 15, 2019

Ellie Morosohk

CFBH-Child and Family Behavioral Health Tampa, FL 33612

RE: Expedited Approval for Initial Review

IRB\#: Pro00040894

Title: Using Generalization-Enhanced Behavioral Skills Training to Teach Poison Safety Skills to Children with Autism

\section{Study Approval Period: 7/15/2019}

Dear Ms. Morosohk:

On 7/15/2019, the Institutional Review Board (IRB) reviewed and APPROVED the above application and all documents contained within, including those outlined below. Please note this study is approved under the 2018 version of 45 CFR 46 and you will be asked to confirm ongoing research annually in place of a full Continuing Review. Amendments and Reportable Events must still be submitted per USF HRPP policy.

\section{Approved Item(s): Protocol Document(s):}

Protocol 440894 Version \#1 7-5-19

\section{Consent/Assent Document(s)*:}

Parental permission \& Parent, Version \#1, 7-12-19.pdf

*Please use only the official IRB stamped informed consent/assent document(s) found under the "Attachments" tab. Please note, these consent/assent documents are valid until the consent document is amended and approved. 
It was the determination of the IRB that your study qualified for expedited review which includes activities that: (1) present no more than minimal risk to human subjects, and (2) involve only procedures listed in one or more of the categories outlined below. The IRB may review research through the expedited review procedure authorized by 45 CFR 46.110. The research proposed in this study is categorized under the following expedited review category:

(6) Collection of data from voice, video, digital, or image recordings made for research purposes.

(7) Research on individual or group characteristics or behavior (including, but not limited to, research on perception, cognition, motivation, identity, language, communication, cultural beliefs or practices, and social behavior) or research employing survey, interview, oral history, focus group, program evaluation, human factors evaluation, or quality assurance methodologies.

This research involving children as participants was approved under 45 CFR 46.404: Research not involving greater than minimal risk to children is presented.

Requirements for Assent and/or Permission by Parents or Guardians: 45 CFR 46.408 Permission of one parent is sufficient.

Assent is waived because it is not appropriate due to the age, maturity, and/or psychological state of the child.

As the principal investigator of this study, it is your responsibility to conduct this study in accordance with IRB policies and procedures and as approved by the IRB. Any changes to the approved research must be submitted to the IRB via an Amendment for review and approval. Additionally, all unanticipated problems must be reported to the USF IRB within five (5) business days.

We appreciate your dedication to the ethical conduct of human subjects research at the University of South Florida and your continued commitment to human research protections. If you have any questions regarding this matter, please call 813-974-5638.

Sincerely,
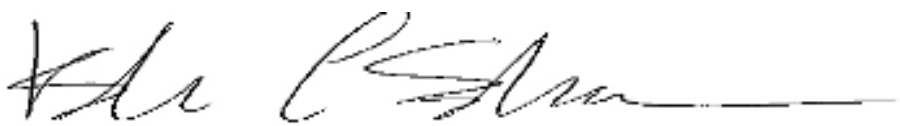

Kristen Salomon, Ph.D., Chairperson USF Institutional Review Board 